\title{
Youth Suicide Prevention across Settings
}

Jo Robinson

Prof. Jo Robinson leads the suicide prevention research unit at Orygen in Melbourne, Australia. Her work focuses on developing and testing novel interventions specifically targeted at vulnerable young people in a range of settings. She is leading a number of studies including the development of the \#chatsafe guidelines, the first evidence-based best practice guidelines for safe peer-peer communication about suicide online, a large-scale school-based study operating across north-west Melbourne, and the establishment of a self-harm monitoring program in emergency departments in Victoria. She also has a keen interest in policy development and has led the development of two major policy reports and is regularly called upon to advise both state and federal governments.

Abstract. Rates of both suicide and self-harm are increasing among young people in Australia and internationally. Despite a growing body of intervention research there is little evidence regarding what does and does not work in youth suicide prevention. A common framework in suicide prevention classifies interventions as universal, selective or indicated. Universal interventions target whole populations regardless of risk; selective interventions target subgroups who may be at elevated risk and indicated interventions target people already displaying suicidal or self-harming behaviour and evidence tells us that interventions should span the full spectrum of approaches. We also know that youth suicide prevention activities need to operate in a range of settings, that reflects the needs and wishes of young people. In this session I will present up to date data on the rates of, and risk factors for, suicide and self-harm in young people. I will then provide a snapshot of work underway in the Youth Suicide Prevention Research Group at Orygen in Melbourne. This will include data from studies that span universal, selective and indicated interventions and that are being conducted in a range of settings, including in health services, schools and on-line. The presentation will conclude with a summary of findings to date and recommendations for ways forward for youth suicide prevention. 